\title{
Connectivity-Based Predictions of Hand Motor Outcome for Patients at the Subacute Stage After Stroke
}

\author{
Julia Lindow ${ }^{1}$, Martin Domin ${ }^{1}$, Matthias Grothe ${ }^{2}$, Ulrike Horn ${ }^{1}$, Simon B. Eickhoff ${ }^{3,4}$ \\ and Martin Lotze ${ }^{1 *}$
}

${ }^{1}$ Functional Imaging Unit, Center for Diagnostic Radiology, University of Greifswald, Greifswald, Germany, ${ }^{2}$ Institute of Neurology, University of Greifswald, Greifswald, Germany, ${ }^{3}$ Institute of Clinical Neuroscience and Medical Psychology, Heinrich Heine University Düsseldorf, Düsseldorf, Germany, ${ }^{4}$ Institute of Neuroscience and Medicine (INM-1), Research Centre Jülich, Jülich, Germany

Background: Connectivity-based predictions of hand motor outcome have been proposed to be useful in stroke patients. We intended to assess the prognostic value of different imaging methods on short-term (3 months) and long-term (6 months) motor outcome after stroke.

Methods: We measured resting state functional connectivity (rsFC), diffusion weighted imaging (DWI) and grip strength in 19 stroke patients within the first days (5-9 days) after stroke. Outcome measurements for short-term (3 months) and long-term (6 months) motor function was assessed by the Motricity Index (MI) of the upper limb and the box and block test (BB). Patients were predominantly mildly affected since signed consent was necessary at inclusion. We performed a multiple stepwise regression analysis to compare the predictive value of rsFC, DWI and clinical measurements.

OPEN ACCESS

Edited by:

Nathalie Tzourio-Mazoyer, CNRS CEA Université Bordeaux, France

Reviewed by: Dong-Hoon Lee, Johns Hopkins University School of Medicine, USA Pavel Lindberg, Université Paris Descartes; U894 INSERM, France

${ }^{*}$ Correspondence: Martin Lotze martin.lotze@uni-greifswald.de

Received: 27 November 2015 Accepted: 25 February 2016 Published: 09 March 2016

Citation: Lindow J, Domin M, Grothe M. Horn U, Eickhoff SB and Lotze M (2016) Connectivity-Based Predictions of Hand Motor Outcome for Patients at the Subacute Stage After Stroke.

Front. Hum. Neurosci. 10:101 doi: 10.3389/fnhum.2016.00101
Results: Patients showed relevant improvement in both motor outcome tests. As expected grip strength at inclusion was a predictor for short- and long-term motor outcome as assessed by Ml. Diffusion-based tract volume (DTV) of the tracts between ipsilesional primary motor cortex and contralesional anterior cerebellar hemisphere showed a strong trend $(p=0.05)$ for a predictive power for long-term motor outcome as measured by MI. DTV of the interhemispheric tracts between both primary motor cortices was predictive for both short- and long-term motor outcome in BB. rsFC was not associated with motor outcome.

Conclusions: Grip strength is a good predictor of hand motor outcome concerning strength-related measurements (MI) for mildly affected subacute patients. Therefore additional connectivity measurements seem to be redundant in this group. Using more complex movement recruiting bilateral motor areas as an outcome parameter, DTV and in particular interhemispheric pathways might enhance predictive value of hand motor outcome.

Keywords: diffusion weighted imaging, motor outcome, prediction, recovery, resting state fMRI, stroke

Abbreviations: BB, Box and Block test; BDI, Beck-Depression-Inventory; cl, contralesional; CST, corticospinal tract; $\mathrm{Cb}$, anterior cerebellar hemisphere; $\mathrm{dPMC}$, dorsal premotor cortex; DTV, diffusion based tract volume; DWI, diffusion weighted imaging; FA, fractional anisotropy; il, ipsilesional; MI, Motricity Index; M1, primary motor cortex; MoCA, Montreal Cognitive Assessment test; NHPT, nine hole peg test; NIHSS, NIH-Stroke-Scale; rsFC, resting state functional connectivity; SMA, supplementary motor area. 


\section{INTRODUCTION}

Stroke is the leading cause of adult disability worldwide, leaving a majority of patients with lingering upper limb impairment (World Health Organization, 2012). Knowing more about motor outcome would be advantageous to achieve the best results in rehabilitation. For long-term motor outcome prediction, parameters are needed that can be assessed within the first days, when patients are in the acute care units.

Functional magnetic resonance imaging (fMRI) raised high expectations because functional representation of movements can be assessed longitudinally in vivo. However, activation fMRI protocols are demanding and patients' compliance is difficult to control for. In contrast, resting state fMRI (rsfMRI) requires little compliance and can therefore be conducted comparable to structural MRI in the acute $(0-24 \mathrm{~h}$ after stroke onset) to subacute ( $24 \mathrm{~h}$ to 6 weeks after stroke) phase after stroke (Di Pino et al., 2014). Especially rs-fMRI functional connectivity (FC) between cortical motor areas has been described to be associated with motor impairment (Carter et al., 2010). Astonishingly, only a low number of studies examined the prognostic value of resting-state functional connectivity (rsFC) for motor outcome in acute stroke patients.

To date the best predictor of later hand motor outcome is the initially measured hand motor impairment. Especially the Fugl-Meyer test (Sanford et al., 1993) has been described as a valuable predictor of hand motor outcome for 2, 6 and 12 months after stroke for mildly to moderately impaired patients (Feys et al., 2000). Likewise the active motion range is known to be a good predictor of short-term (3 months; Beebe and Lang, 2009) and long-term (6 months; Smania et al., 2007) hand motor outcome.

In more severely affected stroke patients, the intactness of the corticospinal tract (CST), as tested with diffusion weighted imaging (DWI) is useful for the prediction of hand motor outcome (Lindenberg et al., 2012; Stinear et al., 2012; Groisser et al., 2014; Byblow et al., 2015). TMSmeasures such as the asymmetry index of hand muscle motor evoked potentials is a clinically well suited predictive method for describing motor outcome in patients after stroke (e.g., Stinear et al., 2012; Byblow et al., 2015). At the subacute phase, a positive association between fractional anisotropy (FA) measured at the height of the posterior limb of the internal capsule of the ipsilesional side and hand motor performance has been shown (Jang et al., 2005; Konishi et al., 2005; Nelles et al., 2008; Byblow et al., 2015). Most authors used FA as well as axial and radial diffusivity for quantification of intactness of the CST. When comparing the predictive value of these measurements, differences in axial diffusivity of the pyramidal tract from the ipsilesional to the contralesional hemisphere at the acute phase had the highest association with 3 and 6 months motor outcome of grip strength and nine hole peg test (NHPT) in a sample of 10 initially strongly impaired stroke patients (Groisser et al., 2014). Probabilistic tractography methods using the DWI data are capable of reconstructing diffusion pathways over long distances even when fibers are crossing (Lindenberg et al., 2010). We thought that the possibility to find alterations among long anatomical pathways might be advantageous for predicting motor outcome even in less severely impaired patients.

Carter et al. (2012a,b) assessed connectivity approaches as a promising method for understanding the impact of cerebral lesions on motor function and its restitution. Consequently, they combined DWI of the CST with FC measurements as assessed by rs-fMRI. For rs-fMRI positive associations with motor performance at the chronic phase after stroke ( $>6$ weeks after stroke; Di Pino et al., 2014) have been described between homotopic motor areas of the affected and the unaffected side indicating that more "balanced" activity between hemispheres is associated with better upper-limb control (Urbin et al., 2014). For rsfMRI two studies describe a positive association between homolog motor areas between both hemispheres and motor performance (Carter et al., 2010, 2012a). However, both did not measure future motor outcome using resting state connectivity but performed only correlative measurements assessed at about the same time. Overall, stroke patients with motor impairment show decreased interhemispheric M1connectiviy and increased resting-state connectivity between ipsilesional M1 and secondary motor areas particularly in the ipsilesional hemisphere (Rehme et al., 2015). Over a period of 3 months the reduced interhemispheric M1 rsFC normalizes (Golestani et al., 2013). To date only one study has applied longitudinal motor outcome measurements to investigate the value of rsFC for predicting motor outcome. Park et al. (2011) investigated rs-fMRI in 12 subacute stroke patients and found a positive association between 6 months motor outcome measured with Fugl-Meyer-Score and rsFC of the ipsilesional M1 with the contralesional thalamus, supplementary motor area (SMA), and medial frontal gyrus.

The present study examined the prognostic value of motor (grip strength, NHPT), and clinical (NIH stroke scale; NIHSS) scores, DWI of long tracts and rs-FC for patients at the subacute stage with predominantly only mild unilateral brain damage. We used two different motor outcome scores: the motricity index (MI) for upper limb and the box and block test (BB) to examine separate aspects of upper limb function namely strength (MI), and hand grip transfer $(\mathrm{BB})$. We hypothesized that intactness of long tracts, would be a predictor for both scores. However, hand strength is represented unilaterally, whereas grip transfer recruits bilateral resources from both hemispheres (Lotze et al., 2012). More bilateral activation might involve increased information transfer (inhibitory or excitatory) via the corpus callosum between both primary motor cortices (M1). Therefore we hypothesized that integrity of interhemispheric fibers would be better predictors for BB, whereas CST integrity might be a better predictor for MI. In addition, we expected lower rsFC between ipsilesional primary motor cortex and contralesional secondary motor areas (SMA, dorsal premotor cortex, dPMC) to be associated 
TABLE 1 | Patient characteristics.

\begin{tabular}{|c|c|c|c|c|c|c|c|c|}
\hline Patient & $\begin{array}{c}\text { Age at } \\
\text { assessment } \\
\text { (years) }\end{array}$ & Gender & $\begin{array}{c}\text { Affected } \\
\text { hemisphere }\end{array}$ & $\begin{array}{l}\text { Lesion } \\
\text { size }\left(\mathrm{cc}^{3}\right)\end{array}$ & $\begin{array}{l}\text { Lesion } \\
\text { location }\end{array}$ & $\begin{array}{c}\text { NIHSS } \\
\text { subacute }\end{array}$ & $\begin{array}{l}\text { Grip strength } \\
\text { subacute } \\
\text { affected } \\
\text { side (bar) }\end{array}$ & $\begin{array}{c}\text { NHPT } \\
\text { subacute } \\
\text { affected side } \\
\text { (pegs/sec) }\end{array}$ \\
\hline 1 & 72 & $\mathrm{~F}$ & L & 0.34 & sc, internal capsule, putamen & 1 & $*$ & 0.33 \\
\hline 2 & 72 & M & $\mathrm{R}$ & 60.63 & c/sc arteria media region & 4 & 0.36 & 0 \\
\hline 3 & 70 & M & $\mathrm{R}$ & 0.27 & sc, internal capsule & 1 & 0.56 & 0.14 \\
\hline 4 & 61 & M & $\mathrm{R}$ & 1.23 & sc, frontal & 1 & 0.76 & 0.34 \\
\hline 7 & 76 & M & $\mathrm{R}$ & 1.31 & sc, internal capsule & 2 & 0.51 & 0.28 \\
\hline 8 & 60 & $\mathrm{~F}$ & $\mathrm{R}$ & 0.06 & sc, frontal & 0 & 0.79 & 0.42 \\
\hline 9 & 70 & $\mathrm{~F}$ & $\mathrm{R}$ & 0.23 & sc, frontal & 1 & 0.53 & 0.44 \\
\hline 10 & 78 & $\mathrm{~F}$ & L & 0.20 & sc, internal capsule & 0 & 0.57 & 0.39 \\
\hline 11 & 72 & $\mathrm{~F}$ & L & 0.21 & sc, pyramidal tract & 2 & 0.50 & 0.35 \\
\hline 12 & 62 & M & $\mathrm{R}$ & 0.16 & sc, frontal & 3 & 0.54 & 0.39 \\
\hline 17 & 74 & M & L & 1.18 & sc, pyramidal tract & 1 & 0.73 & 0.38 \\
\hline 18 & 64 & $\mathrm{~F}$ & $L$ & 0.56 & sc, int. capsule & 3 & 0.58 & 0.26 \\
\hline 19 & 49 & M & $\mathrm{L}$ & 0.47 & sc, int. capsule, putamen & 2 & 0.42 & 0.19 \\
\hline Mean & 69.67 & $8 \mathrm{~F} / 11 \mathrm{M}$ & $9 \mathrm{~L} / 10 \mathrm{R}$ & 5.18 & & 1.63 & 0.62 & 0.34 \\
\hline
\end{tabular}

Abbreviations: F, female; M, male; L, left; R, right; NIHSS subacute measured during the first 5 days; c, cortical; sc, subcortical; int. capsule: internal capsule. *Measurement not possible because of initial injury.

with better motor outcome (MI and BB; Wang et al., 2010).

\section{MATERIALS AND METHODS}

\section{Participants}

Twenty-four stroke patients [aged $67.7 \pm 8.7$ years (mean \pm standard deviation), 11 female, 19 right handed] were recruited through the stroke unit at the Department of Neurology of the University Medicine Greifswald.

Inclusion criteria were: (1) first ischemic stroke; (2) unilateral upper limb impairment at day 2 after stroke; (3) no contraindications for MRI; (4) older than 18 years; and (5) being able to consent for study participation at day 5 after stroke. Exclusion criteria were: (1) global aphasia; (2) cognitive impairment; and (3) other neurological diseases. Overall, 19 patients were included in the 3 months survey and 17 in the 6 months survey (see Table 1 and Figure 1).

All subjects gave written informed consent to attend the study, which was approved by the National Ethics Committee of the German Society for Psychology (DPG).

\section{Clinical Measures}

Stroke severity was determined by the National Institutes of Health Stroke Scale (NIHSS) that ranges from 0 (no deficits) to 42 (severe stroke) at the time of hospitalization (NIHSS initial), at inclusion (NIHSS subacute), at 3 months (NIHSS 3 months) and 6 months (NIHSS 6 months) post-stroke (Brott et al., 1989).
The "Montreal Cognitive Assessment Test" (MoCA) was used to screen for cognitive abilities in various domains: visuospatial and short-term memory, attention, language, abstraction, executive function, and orientation (Dong et al., 2010). The BeckDepression-Inventory (BDI) was used to screen for depression (Beck et al., 1961). These global scores revealed that patients on average were moderately depressed $(7.57 \pm 9.41)$ post stroke and showed mild cognitive impairment (MoCA index after 3 months: $22.84 \pm 3.30)$.

To investigate the predictive value of motor scores we tested two measurements of hand motor performance initially, 3 and 6 months post-stroke: hand grip strength (Martin Vigorimeter, Gebrüder Martin, Tuttlingen, Germany) and finger dexterity (nine hole peg test, NHPT). For grip strength the average value of five trials was taken as the value for grip strength for each hand (Desrosiers et al., 1995). For finger dexterity, we used the NHPT, measuring the time to put all pins into the nine holes and remove them back into the container. The NHPT score was determined by averaging over two trials (Mathiowetz et al., 1985a). Results were expressed in pegs per second (higher value means better performance).

As motor outcome variables we used the MI motricity index of the affected upper limb and the BB (Mathiowetz et al., 1985b). The MI is especially known for being a valid instrument to assess the strength of the paretic upper extremity after stroke by measuring the degree of the paresis including hand-grasp, elbow flexion and shoulder abduction (Collin and Wade, 1990). It has been applied in several studies on motor outcome in stroke patients (Kwakkel et al., 2006). The score 


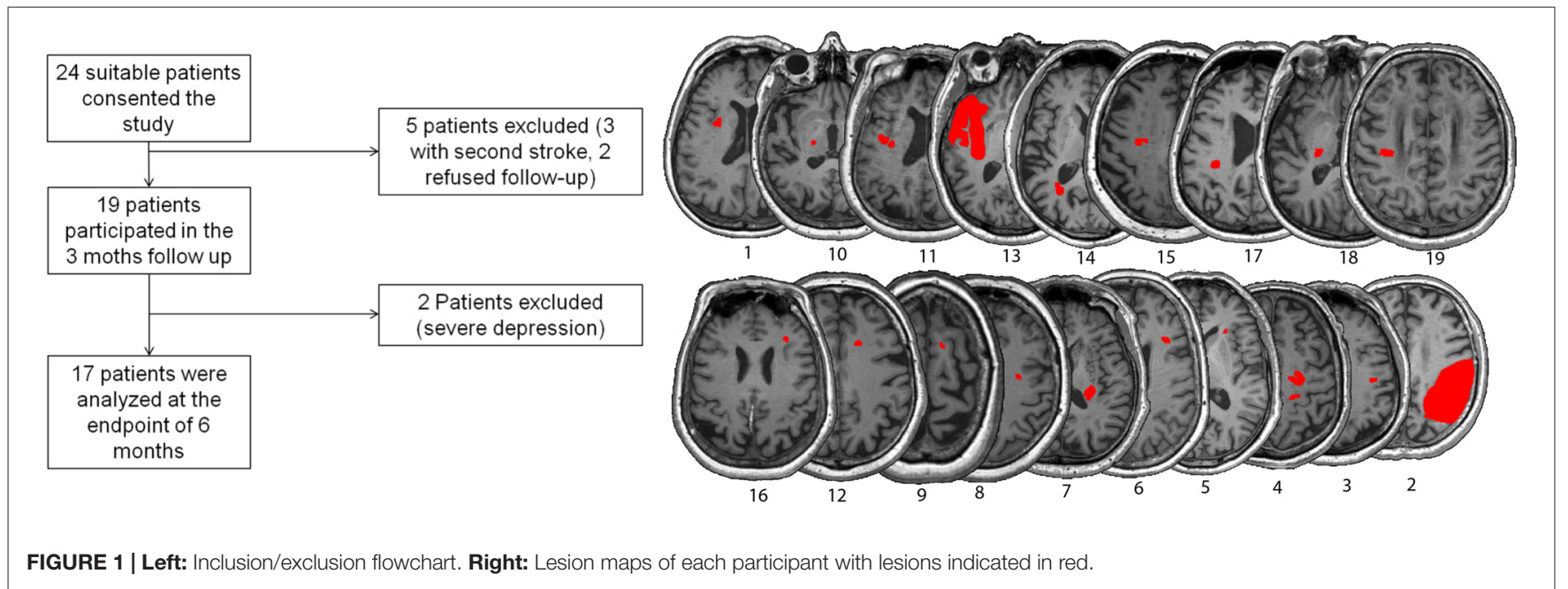

ranges from minimum score of 0 (plegic) to the maximum of 100 (recovered). MI was acquired at baseline (subacute stage), 3 and 6 months after stroke. The $\mathrm{BB}$ is a valid measurement to determine unilateral gross manual dexterity (Platz et al., 2005). Complex movements of several joints are required to move as many blocks as possible from one compartment to another within $60 \mathrm{~s}$. The $\mathrm{BB}$ was acquired at 3 and 6 months after stroke. The average number of cube transferred per minute within the age range of patients in this study is 69 .

\section{Duration of Neurorehabilitation}

We assessed the following information about the Neurorehabilitation: all patients got physical and/or occupational therapy during their hospital stay, whose average length was $9.3 \pm 3.6$ days. Afterwards 11 patients (64.7\%) were sent to an acute neurorehabilitation clinic and the average days of therapy were $27.12 \pm 11.6$ days. Additionally five patients (29.4\%) had an ambulant physical and/or occupational therapy when they were back at home again with an average of $22.4 \pm 24.3 \mathrm{~h}$. Overall, the total number of physical and/or occupational therapy days that the patient received between the hospital stay and the 6 months follow-up amounted to $27.7 \pm 11.4$ days.

\section{Magnetic Resonance Imaging (MRI)}

MRI was conducted with a 3 Tesla MRI-Scanner (Verio, Siemens, Erlangen, Germany) using a 32-channel head coil from day 5 after stroke (average: 7.5 days) to avoid artifact from tissue edema affecting water diffusion. After day 5 the effect of edema was assumed to be negligible (Sotak, 2002).

\section{Resting State fMRI}

We used gradient echo planar imaging (EPI) of 150 whole head volumes with 36 transverse slices each, with an in plane spatial resolution of $3 \times 3 \mathrm{~mm}^{2}$, slice thickness of 3 and $1 \mathrm{~mm}$ gap. The temporal resolution (TR) of each volume was 2 s. The whole rs-fMRI lasted for a period of
6 min. Participants were instructed to close their eyes, let their mind wander and avoid to mentally fixate on a certain issue.

\section{T1-Weighted Cranial Imaging}

An anatomical 3D T1-weighted MPRage dataset was acquired with a voxel size of $1 \mathrm{~mm}$ isotropic, acquiring 176 sagittal slices, with a TE of $2.5 \mathrm{~ms}$ and a TR of $1900 \mathrm{~ms}$. The whole measurement duration was about $7 \mathrm{~min}$.

\section{Diffusion Weighted Imaging}

We applied a Siemens MDDW (Multi Directional Diffusion Weighting) sequence with the following parameter setup: voxel size: $1.8 \times 1.8 \times 2.3 \mathrm{~mm}^{3}, 55$ slices, 1 acquisition and 64 directions. One b0-volume was measured and $b=1000 \mathrm{~s} / \mathrm{mm}^{2}$ was used for the diffusion-weighted images. TR was $10,500 \mathrm{~ms}$, TE: $107 \mathrm{~ms}$ and the total scan time was $12 \mathrm{~min}$.

\section{Data Evaluation}

\section{DWI Processing}

Gray matter parts of all cortical ROIs (MNI-space) were removed by using a white matter mask (MNI-space, provided by FSL) in order to prevent tractography to and from non-white matter structures. DWI data were coregistered to the anatomical $3 \mathrm{D}$ T1-weighted dataset, which in turn was spatially normalized to the MNI space using FSL FNIRT. The inverse of the resulting transformation was used to spatially denormalize the masked ROIs into subject space. DWI data were processed utilizing BEDPOSTX and PROBTRACKX of the FSL software package. First, the diffusion data were corrected for eddy current and head movement artifacts. Then, BEDPOSTX was executed to build up distributions on diffusion parameters and modeling crossing fibers at each voxel of the brain. PROBTRACKX was used to calculate a structural connectivity distribution between selected ROIs (Behrens et al., 2007). The resulting probabilistic streamlines were then normalized by region-of-interest sizes (volume of all contained voxels; Rilling et al., 2008) and thresholded with $10 \%$ of the highest connectivity value of a 

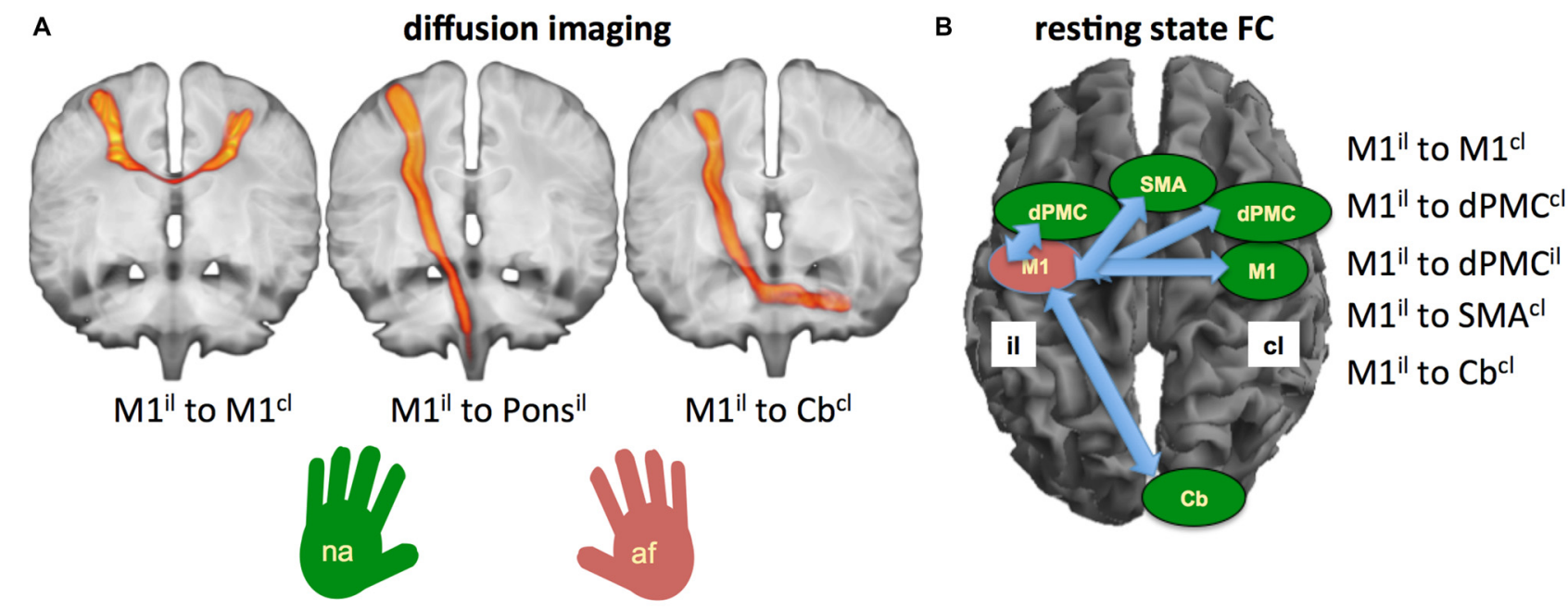

FIGURE 2 | Visualization of tracts and regions of interest (ROIs) used for diffusion-based tract volume (DTV) and rsFC analysis. (A) Pathways included

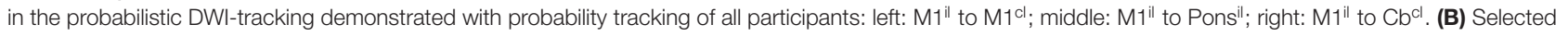
ROls to calculate rsFC. Abbreviations: M1, primary motor cortex; dPMC, dorsal premotor cortex; SMA, supplementary motor area; Cb, cerebellum; il, ipsilesional; cl, contralesional; af, affected hand; na, non-affected hand.

certain probabilistic streamline. The normalized and thresholded probabilistic streamline was evaluated with regard to its voxel volume. This value is now referred to as diffusion-based tract volume (DTV).

\section{Resting State Preprocessing}

Data were preprocessed using SPM8 (Wellcome Department of Cognitive Neuroscience, London, UK). Images were first corrected for head movement by affine registration using a two-pass procedure by which images were initially realigned to the first image and subsequently to the mean of the realigned images. Each participant's mean image was then spatially normalized to the Montreal Neurological Institute (MNI) single-subject template brain using the "unified segmentation" approach and the ensuing deformation was applied to the individual EPI volumes (Ashburner and Friston, 2005). Hereby, volumes were resampled at $1.5 \times 1.5 \times 1.5 \mathrm{~mm}^{3}$ voxel size. Images were then smoothed by a $5 \mathrm{~mm}$ full-width at half-maximum Gaussian kernel to increase the signalto-noise ratio and compensate for remaining differences in individual anatomy. Confound removal was performed as described before Lotze et al. (2014). After confound removal, data were band-pass filtered preserving frequencies between 0.01 and $0.08 \mathrm{~Hz}$, as meaningful resting-state correlations will predominantly be found in these frequencies given that the BOLD response acts as a low-pass fimoot (Greicius et al., 2003).

\section{Regions of Interest (ROIs)}

We included the following ROIs: the primary motor cortex (M1) both ipsilesional (il) and contralesional (cl) as seeds for both rsFC and DTV (Carter et al., 2010, 2012a; Lindenberg et al., 2010; Lu et al., 2011; Urbin et al., 2014). In addition, we tested the long pathways from $\mathrm{M}^{\mathrm{il}}$ to the anterior cerebellar hemisphere ${ }^{\mathrm{cl}}$ (cerebellar Larsell lobules H IV-VI; $\mathrm{Cb}^{\mathrm{cl}}$ ) for both the rsFC and the DTV-analysis (for rsFC: Wang et al., 2010; Lu et al., 2011; for DWI: Groisser et al., 2014). The dPMC and SMA proper were only used as seeds for rsFC (Carter et al., 2010, 2012a; Rehme et al., 2011). In contrast, pathways descending from M1 to the height of the pons have been only included for the DTV-analysis (see also Lindenberg et al., 2010).

For our study APMC and SMA proper were selected from the HMAT-Atlas (Human Motor Area Template, Laboratory for Rehabilitation Neuroscience, University of Florida). The seed for the primary hand motor area was generated by drawing a point into the hand knob area of both the left and right side of the brain (Yousry et al., 1997). The point represented the center for a spherical region of $7 \mathrm{~mm}$ radius, which was created by a dilation process. The JHU ICBM-DTI-81 White-Matter Labels (Laboratory of Brain Anatomical MRI, Johns Hopkins University) were used to extract ROIs for the descending pyramidal tract in the ipsilesional pons.

We applied probabilistic tractography and did not use conventional diffusion tractography (DTI) quantification (FA or diffusivity) since probabilistic tractography is better suited for following white matter connections over long distances $\left(\mathrm{M} 1^{\text {il }}\right.$ with the pons ${ }^{\mathrm{il}}, \mathrm{M}^{\mathrm{il}}$ with the anterior cerebellar hemisphere $\left.{ }^{\mathrm{cl}}\right)$ or between the primary motor cortices $\left(\mathrm{M}^{\mathrm{il}}-\mathrm{M} 1^{\mathrm{cl}}\right.$; Bucci et al., 2013).

For each participant, the rsFC time-series data of each seed region were extracted and correlated with each other, and the resulting Pearson correlation coefficients were transformed into Fisher's $Z$ scores. These were then used for further statistical testing. 


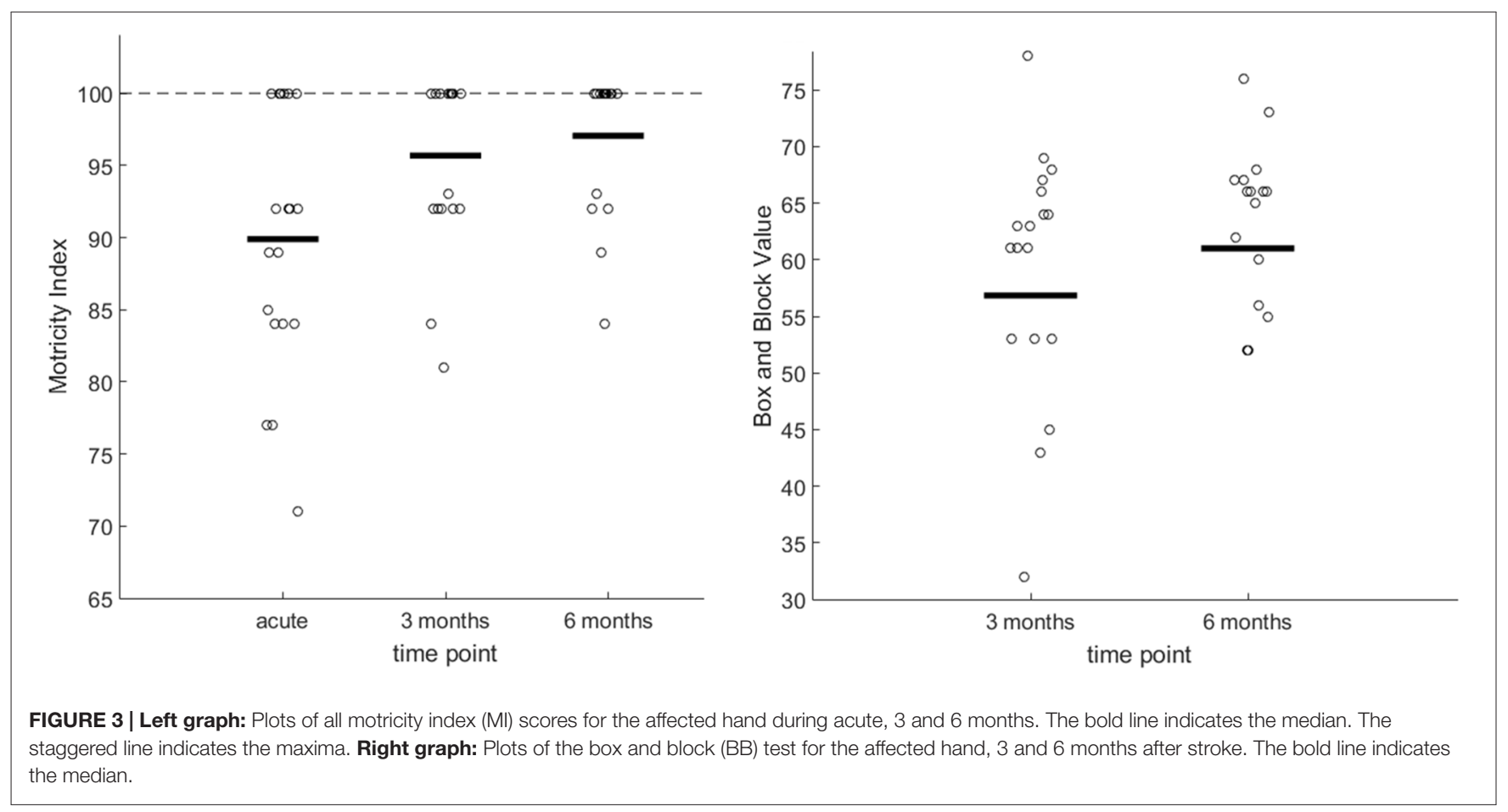

\section{Statistical Testing}

We used Wilcoxon tests to determine differences between motor scores (MI and $\mathrm{BB}$ ) between time points. The predictive value for the outcome of the MI and $\mathrm{BB}$ of the affected hand at 3 and 6 months was calculated in six stepwise multiple linear regression analyses of parameters assessed in the subacute phase. These comprised clinical scores (grip strength, NIHSS and NHPtest), probabilistic tracking $\left(\mathrm{Mi}^{\mathrm{il}}-\mathrm{M} 1^{\mathrm{cl}}, \mathrm{M}^{\mathrm{il}}-\right.$ pons $\left.{ }^{\mathrm{il}}, \mathrm{M}^{\mathrm{il}}-\mathrm{Cb}^{\mathrm{cl}}\right)$ and rsFC $\left(\mathrm{Mi}^{\mathrm{il}}-\mathrm{M}^{\mathrm{cl}}\right.$; $\mathrm{M1}^{\mathrm{il}}-\mathrm{dPMC} \mathrm{C}^{\mathrm{cl}}, \mathrm{M}^{\mathrm{il}}-\mathrm{dPMC}{ }^{\mathrm{il}}$; $\mathrm{M}^{\mathrm{il}}-\mathrm{SMA}^{\mathrm{cl}}$, $\mathrm{Ml}^{\mathrm{il}}-\mathrm{Cb}^{\mathrm{cl}}$ ) for the two time points each (3 and 6 months; see Figure 2).

The goodness of fit is expressed in terms of $R^{2}$ and significance in an F-test. The resulting standardized regression coefficients are given. Table 2 provides an overview on the models tested and the results.

\section{RESULTS}

The NIHSS score at admission was $5.32 \pm 5.25$ and $1.63 \pm 1.01$ at time of MRI measurement (average: 7.5 days). MI showed relevant changes during the short term [Wilcoxon test between baseline and 3 months: $U_{(18)}=2.51 ; p=0.012$ ] and long term period [between 3 and 6 months: $U_{(16)}=1.73 ; p=0.040$; see plots in Figure 3, left]. BB of the affected hand at 3 months was on average $56.84 \pm 14.53$ and at 6 months $61.00 \pm 13.26$ (see plots in Figure 4, right) with significant differences between 3 and 6 months measures $\left(U_{(16)}=2.28\right.$; $p=0.023)$.

Grip strength predicted MI after 3 months $\left(R^{2}=0.54 ; p=\right.$ $0.001)$ and 6 months $\left(R^{2}=0.44 ; p=0.008\right.$; Figure 4). NHPT and NIHSS were not suited to predict motor outcome and were discarded by the stepwise regression. In contrast, no motor outcome measurement (NIHSS, NHPT, MI), was associated significantly with $\mathrm{BB}$.

DTV showed a very weak predictive value for both short-term and long-term motor outcome. For the 6-month MI outcome we found a strong trend for a predictive value for diffusion probability between $\mathrm{M}^{\text {il }}$ and $\mathrm{Cb}^{\mathrm{cl}}\left(R^{2}=0.23 ; p=0.05\right)$. For the $\mathrm{BB}$, we found both a short-term ( 3 months: $R^{2}=0.25 ; p=$ $0.03)$ and a long- term ( 6 month: $\left.R^{2}=0.28 ; p=0.03\right)$ predictive value for the $\mathrm{M} 1^{\text {il }}-\mathrm{M} 1^{\mathrm{cl}} \mathrm{DTV}$ tract probability (Figure 4). Table 2 provides an overview on the comparisons and their strength of association. All other DTV-values were rated as irrelevant by multiple regression analyses.

TABLE 2 | Prediction of motor outcome parameters with stepwise multiple linear regression.

\begin{tabular}{|c|c|c|}
\hline \multirow[t]{2}{*}{ Predictive values } & \multicolumn{2}{|c|}{ Outcome parameter } \\
\hline & MI 3 months & MI 6 months \\
\hline Clinical measures & $\begin{array}{l}\text { Grip strength } \\
\left(R^{2}=0.54\right)\end{array}$ & $\begin{array}{l}\text { Grip strength } \\
\left(R^{2}=0.44\right)\end{array}$ \\
\hline Resting state & No predictive power & No predictive power \\
\hline DWI & No predictive power & $\begin{array}{l}\text { Mil-ant cerebell hem }^{\mathrm{c}} \\
\left(R^{2}=0.23\right)\end{array}$ \\
\hline & BB 3 months & BB 6 months \\
\hline Clinical measures & No predictive power & No predictive power \\
\hline Resting state & No predictive power & No predictive power \\
\hline DWI & $\begin{array}{l}\mathrm{M} 1^{\mathrm{il}}-\mathrm{M} 1^{\mathrm{cl}} \\
\left(R^{2}=0.25\right)\end{array}$ & $\begin{array}{l}\mathrm{M} 1^{\mathrm{il}}-\mathrm{M} 1^{\mathrm{cl}} \\
\left(R^{2}=0.28\right)\end{array}$ \\
\hline
\end{tabular}

Abbreviations: MI, motricity index; $B B$, block and box test; DWI, diffusion weighted imaging; M1, primary motor cortex; il, ipsilesional; cl, contralesional. 


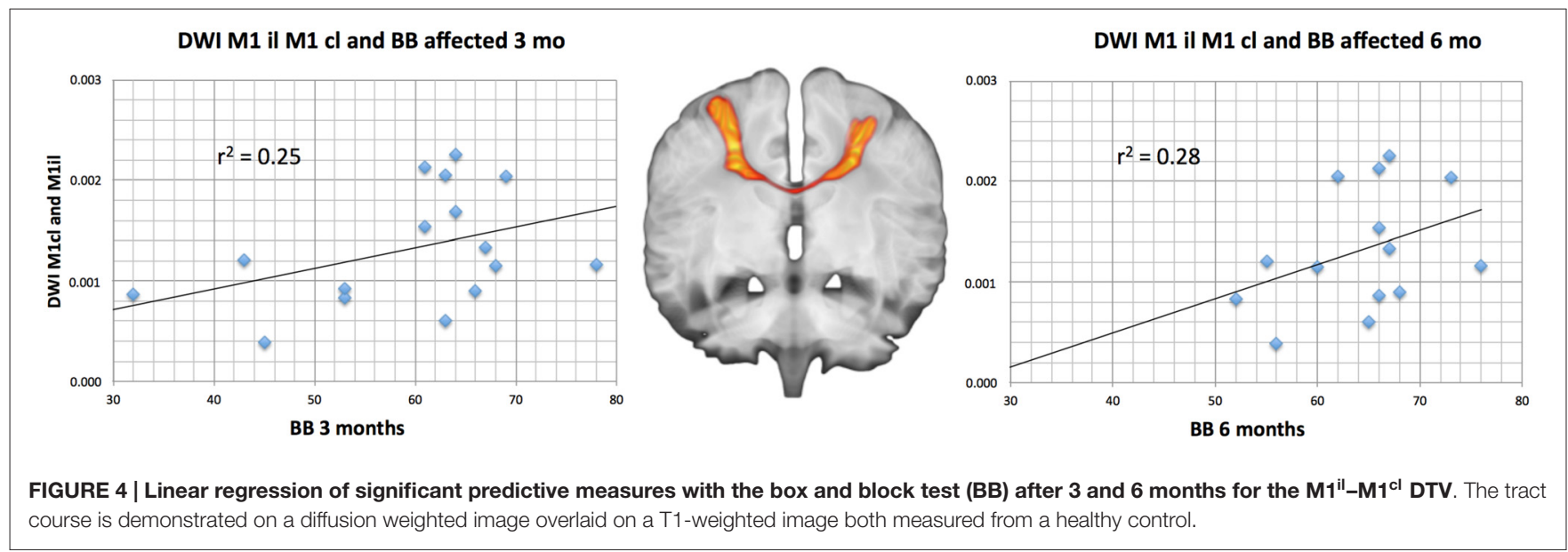

None of the rsFC values between the seeds showed any association with later motor outcome for $\mathrm{MI}$ or $\mathrm{BB}$ and were discarded by the stepwise regression.

\section{DISCUSSION}

In this study, we tested the predictive value of motor scores (grip strength, NHPT), the NIHSS, rsFC and DTV for shortand long-term motor outcome in subacute stroke patients with predominantly mild cerebral damage. Initial grip strength was predictive of MI. The intactness of interhemispheric tracts was predictive of $\mathrm{BB}$ outcome. In contrast, cerebrospinal pathways from ipsilesional M1 to contralesional anterior cerebellar hemisphere were most important for MI outcome prediction rsFC showed no predictive value.

\section{The Predictive Value of Motor Assessments}

Our study confirms earlier reports that strength measurements during the subacute stage have a predictive value for MI in initially mildly affected patients (Smania et al., 2007; Beebe and Lang, 2009). However, a high ceiling effect of MI already after 3 months due to the mild initial impairment in our patients restricted the usefulness of MI as an outcome variable. When predicting $\mathrm{BB}$ as an outcome measurement, there were no associations for strength, pinch grip or NIHSS. The above finding illustrates the importance of outcome measures for different aspects of motor performance. Aspects explained by one variable might not necessarily explain others.

\section{The Predictive Value of DTV}

In our study, both the DTV for the long CST between ipsilesional M1 and the contralesional anterior cerebellar hemisphere and the M1 connecting interhemispheric tract showed relevant predictive value for motor outcome. It is not surprising that corticospinal integrity of the pyramidal tract form ipsilesional M1 is relevant for motor outcome. For severely affected patients, it has been demonstrated that intactness of the CST (van Dokkum et al., 2014) is a strong predictor for resolution of impairment (Byblow et al., 2015; Burke Quinlan et al., 2015) and can be used within an algorithm (Stinear et al., 2012) to inform about the potential of upper limb functional recovery. However, probabilistic tracking only for the most distant seeds (M1 to cerebellar hemisphere) showed any predictive value in our subacute stroke patients with predominantly mildly damage. This is certainly caused by the small amount of lesions observed in our patients leaving them with mild motor impairment. The intactness of the CST can be assessed with axonal or radial diffusivity (van Dokkum et al., 2014) as well as with FA (Schulz et al., 2012) within the posterior limb of the internal capsule. FA is a rather global measure since tracts from the $\mathrm{APMC}$, the SMA and M1 and S1 and parietal cortex are passing through this structure and especially those descending from M1 and dPMC correlate positively with grip strength in the chronic stage (Schulz et al., 2012). Probabilistic tractography is capable of testing long distance white matter tracts and is well suited for patients without circumscribed lesions to the pyramidal tract.

White matter integrity of non-crossing fibers between M1-M1 can predict training-induced performance gains in chronic patients (Lindenberg et al., 2012). Our findings are in accordance with this earlier result. We found a predictive value of $\mathrm{M}^{\mathrm{il}}-\mathrm{M} 1^{\mathrm{cl}}$ diffusivity for short- and long-term outcome of the BB. Especially for tasks recruiting bilateral resources such as during grasping and the transfer of objects, these interhemispheric tracts are of importance since they enable a bihemispheric coordination of sensorimotor (SM1) activation (Lotze et al., 2012).

\section{The Predictive Value of rsFC}

In our study, rsFC had no predictive value for early or late outcomes spanning the subacute stage. For patients with motor impairment after stroke a decreased $\mathrm{rsFC}$ between $\mathrm{M} 1^{\text {il }}$ and $\mathrm{M}^{\mathrm{cl}}$ in comparison to healthy age-matched controls is the most consistent finding reported in resting state studies on stroke patients (Carter et al., 2010, 2012a; Wang et al., 2010; Park et al., 2011; Golestani et al., 2013; Rehme et al., 2015). 
Previous studies measuring rsFC at the subacute stage found significant associations with motor performance at time of fMRI (Carter et al., 2010, 2012a). This however has no predictive value as demonstrated in our study using longitudinal performance measurements.

At least four longitudinal rs-fMRI studies have been conducted with stroke patients (Wang et al., 2010; Lu et al., 2011; Park et al., 2011; Golestani et al., 2013). Golestani et al. (2013) investigated 31 stroke patients with motor impairment within the first $24 \mathrm{~h}$, after 7 and after 90 days and found that over time the reduced interhemispheric SM1 rsFC normalized. Their work is an excellent example of how variable rsFC is after stroke. This may be the reason why long-term motor outcome prediction is problematic using this measurement. In addition, a very recent study demonstrated that highest intra-participant variability was observed over extensive repetitive measurements in a healthy participant in the somato-motor regions (Laumann et al., 2015). This low intra-subject reliability may indicate a poor prognostic value.

\section{Limitations}

Sample size is a limitation of the present study. Combining data from separate centers would be necessary to overcome this problem. It might further be advantageous to differentiate cortical and subcortical damage in a larger sample. Another limitation was the selection of patients who were mainly mildly to moderately impaired. Inclusion of more patients with severe initial impairment may produce different results but was excluded by our ethical commitment. Another limitation was that we could not perform BB at the subacute stage for reason of hygiene in an intensive care unit. In addition, it can not be excluded that edema at the early phase after stroke does affect imaging results.

\section{REFERENCES}

Ashburner, J., and Friston, K. J. (2005). Unified segmentation. Neuroimage 26, 839-851. doi: 10.1016/j.neuroimage.2005.02.018

Beck, A. T., Ward, C. H., Mendelson, M., Mock, J., and Erbaugh, J. (1961). An inventory for measuring depression. Arch. Gen. Psychiatry 4, 561-571. doi: 10. 1001/archpsyc.1961.01710120031004

Beebe, J. A., and Lang, C. E. (2009). Active range of motion predicts upper extremity function 3 months after stroke. Stroke 40, 1772-1779. doi: 10. 1161/STROKEAHA.108.536763

Behrens, T. E. J., Berg, H. J., Jbabdi, S., Rushworth, M. F. S., and Woolrich, M. W. (2007). Probabilistic diffusion tractography with multiple fibre orientations: what can we gain? Neuroimage 34, 144-155. doi: 10.1016/j.neuroimage.2006. 09.018

Brott, T., Adams, H. P., Olinger, C. P., Marler, J. R., Barsan, W. G., Biller, J., et al. (1989). Measurements of acute cerebral infarction: a clinical examination scale. Stroke 20, 864-870. doi: 10.1161/01.str.20.7.864

Bucci, M., Mandelli, M. L., Berman, J. I., Amirbekian, B., Nguyen, C., Berger, M. S., et al. (2013). Quantifying diffusion MRI tractography of the corticospinal tract in brain tumors with deterministic and probabilistic methods. Neuroimage Clin. 3, 361-368. doi: 10.1016/j.nicl.2013.08.008

Burke Quinlan, E., Dodakian, L., See, J., McKenzie, A., Le, V., Wojnowicz, M., et al. (2015). Neural function, injury, and stroke subtype predict treatment gains after stroke. Ann. Neurol. 77, 132-145. doi: 10.1002/ana.24309

\section{CONCLUSION}

rsFC is strongly variable after stroke what makes any motor outcome prediction for this parameter difficult. It is quite astonishing that a predictive value of resting state connectivity has been postulated without performing longitudinal measurements. It might also be useful to explore the usefulness of including probabilistic tractography in combined outcome algorithms (Stinear et al., 2012, 2013, 2014). However, any potential improvements in prognosis would have to be weighed up against any added complexity of more advanced techniques. A simple approach may be necessary for optimal application in clinical practice.

\section{AUTHOR CONTRIBUTIONS}

JL performed measurements and investigations on the patients and contributed in writing the manuscript. MD evaluated and interpreted the DWI-data. MG helped with patient recruitment and with interpretation of data. UH helped with statistical evaluation. SBE provided tools for performing the resting state analysis and commented the analysis and the manuscript. ML planned the study, performed the statistics and wrote the manuscript.

\section{FUNDING}

The study was funded by a grant from the German Research Community (DFG Lo795/7-1).

\section{ACKNOWLEDGMENTS}

We would like to thank Winston D. Byblow for valuable advice in conducting this study.

Byblow, W. D., Stinear, C. M., Barber, P. A., Petoe, M. A., and Ackerley, S. J. (2015). Proportional recovery after stroke depends on corticomotor integrity. Ann. Neurol. 78, 848-859. doi: 10.1002/ana.24472

Carter, A. R., Astafiev, S. V., Lang, C. E., Connor, L. T., Rengachary, J., Strube, M. J., et al. (2010). Resting interhemispheric functional magnetic resonance imaging connectivity predicts performance after stroke. Ann. Neurol. 67, 365-375. doi: 10.1002/ana.21905

Carter, A. R., Patel, K. R., Astafiev, S. V., Snyder, A. Z., Rengachary, J., Strube, M. J., et al. (2012a). Upstream dysfunction of somatomotor functional connectivity after corticospinal damage in stroke. Neurorehabil. Neural Repair 26, 7-19. doi: $10.1177 / 1545968311411054$

Carter, A. R., Shulman, G. L., and Corbetta, M. (2012b). Why use a connectivitybased approach to study stroke and recovery of function? Neuroimage 62 , 2271-2280. doi: 10.1016/j.neuroimage.2012.02.070

Collin, C., and Wade, D. (1990). Assessing motor impairment after stroke: a pilot reliability study. J. Neurol. Neurosurg. Psychiatry 53, 576-579. doi: 10. 1136/jnnp.53.7.576

Desrosiers, J., Hébert, R., Bravo, G., and Dutil, E. (1995). Comparison of the Jamar dynamometer and the Martin vigorimeter for grip strength measurements in a healthy elderly population. Scand. J. Rehabil. Med. 27, 137-143.

Di Pino, G., Pellegrino, G., Assenza, G., Capone, F., Ferreri, F., Formica, D., et al. (2014). Modulation of brain plasticity in stroke: a novel model for neurorehabilitation. Nat. Rev. Neurol. 10, 597-608. doi: 10.1038/nrneurol. 2014.162 
Dong, Y., Sharma, V. K., Chan, B. P.-L., Venketasubramanian, N., Teoh, H. L., Seet, R. C. S., et al. (2010). The Montreal Cognitive Assessment (MoCA) is superior to the Mini-Mental State Examination (MMSE) for the detection of vascular cognitive impairment after acute stroke. J. Neurol. Sci. 299, 15-18. doi: 10.1016/j.jns.2010.08.051

Feys, H., De Weerdt, W., Nuyens, G., van de Winckel, A., Selz, B., and Kiekens, C. (2000). Predicting motor recovery of the upper limb after stroke rehabilitation: value of a clinical examination. Physiother. Res. Int. 5, 1-18. doi: 10.1002/ pri. 180

Golestani, A.-M., Tymchuk, S., Demchuk, A., Goodyear, B. G., and VISION-2 Study Group. (2013). Longitudinal evaluation of resting-state FMRI after acute stroke with hemiparesis. Neurorehabil. Neural Repair 27, 153-163. doi: 10. $1177 / 1545968312457827$

Greicius, M. D., Krasnow, B., Reiss, A. L., and Menon, V. (2003). Functional connectivity in the resting brain: a network analysis of the default mode hypothesis. Proc. Natl. Acad. Sci. U S A 100, 253-258. doi: 10.1073/pnas. 0135058100

Groisser, B. N., Copen, W. A., Singhal, A. B., Hirai, K. K., and Schaechter, J. D. (2014). Corticospinal tract diffusion abnormalities early after stroke predict motor outcome. Neurorehabil. Neural Repair 28, 751-760. doi: 10. $1177 / 1545968314521896$

Jang, S. H., You, S. H., Kwon, Y.-H., Hallett, M., Lee, M. Y., and Ahn, S. H. (2005). Cortical reorganization associated lower extremity motor recovery as evidenced by functional MRI and diffusion tensor tractography in a stroke patient. Restor. Neurol. Neurosci. 23, 325-329.

Konishi, J., Yamada, K., Kizu, O., Ito, H., Sugimura, K., Yoshikawa, K., et al. (2005). MR tractography for the evaluation of functional recovery from lenticulostriate infarcts. Neurology 64, 108-113. doi: 10.1212/01.wnl.0000148477.65273.0c

Kwakkel, G., Kollen, B., and Twisk, J. (2006). Impact of time on improvement of outcome after stroke. Stroke 37, 2348-2353. doi: 10.1161/01.str.0000238594. 91938.1e

Laumann, T. O., Gordon, E. M., Adeyemo, B., Snyder, A. Z., Joo, S. J., Chen, M.Y., et al. (2015). Functional system and areal organization of a highly sampled individual human brain. Neuron 87, 657-670. doi: 10.1016/j.neuron.2015. 06.037

Lindenberg, R., Renga, V., Zhu, L. L., Betzler, F., Alsop, D., and Schlaug, G. (2010). Structural integrity of corticospinal motor fibers predicts motor impairment in chronic stroke. Neurology 74, 280-287. doi: 10.1212/WNL.0b013e3181ccc6d9

Lindenberg, R., Zhu, L. L., Rüber, T., and Schlaug, G. (2012). Predicting functional motor potential in chronic stroke patients using diffusion tensor imaging. Hum. Brain Mapp. 33, 1040-1051. doi: 10.1002/hbm. 21266

Lotze, M., Beutling, W., Loibl, M., Domin, M., Platz, T., Schminke, U., et al. (2012). Contralesional motor cortex activation depends on ipsilesional corticospinal tract integrity in well-recovered subcortical stroke patients. Neurorehabil. Neural Repair 26, 594-603. doi: 10.1177/1545968311 427706

Lotze, M., Erhard, K., Neumann, N., Eickhoff, S. B., and Langner, R. (2014). Neural correlates of verbal creativity: differences in resting-state functional connectivity associated with expertise in creative writing. Front. Hum. Neurosci. 8:516. doi: 10.3389/fnhum.2014.00516

Lu, J., Liu, H., Zhang, M., Wang, D., Cao, Y., Ma, Q., et al. (2011). Focal pontine lesions provide evidence that intrinsic functional connectivity reflects polysynaptic anatomical pathways. J. Neurosci. 31, 15065-15071. doi: 10. 1523/JNEUROSCI.2364-11.2011

Mathiowetz, V., Kashman, N., Volland, G., Weber, K., Dowe, M., and Rogers, S. (1985a). Grip and pinch strength: normative data for adults. Arch. Phys. Med. Rehabil. 66, 69-74.

Mathiowetz, V., Volland, G., Kashman, N., and Weber, K. (1985b). Adult norms for the box and block test of manual dexterity. Am. J. Occup. Ther. 39, 386-391. doi: 10.5014/ajot.39.6.386

Nelles, M., Gieseke, J., Flacke, S., Lachenmayer, L., Schild, H. H., and Urbach, H. (2008). Diffusion tensor pyramidal tractography in patients with anterior choroidal artery infarcts. AJNR Am. J. Neuroradiol. 29, 488-493. doi: 10. 3174/ajnr.a0855

Park, C., Chang, W. H., Ohn, S. H., Kim, S. T., Bang, O. Y., Pascual-Leone, A., et al. (2011). Longitudinal changes of resting-state functional connectivity during motor recovery after stroke. Stroke 42, 1357-1362. doi: 10.1161/STROKEAHA. 110.596155
Platz, T., Pinkowski, C., van Wijck, F., Kim, I.-H., di Bella, P., and Johnson, G. (2005). Reliability and validity of arm function assessment with standardized guidelines for the Fugl-Meyer test, action research arm test and box and block test: a multicentre study. Clin. Rehabil. 19, 404-411. doi: 10. 1191/0269215505cr832oa

Rehme, A. K., Fink, G. R., von Cramon, D. Y., and Grefkes, C. (2011). The role of the contralesional motor cortex for motor recovery in the early days after stroke assessed with longitudinal FMRI. Cereb. Cortex 21, 756-768. doi: 10. 1093/cercor/bhq140

Rehme, A. K., Volz, L. J., Feis, D.-L., Bomilcar-Focke, I., Liebig, T., Eickhoff, S. B., et al. (2015). Identifying neuroimaging markers of motor disability in acute stroke by machine learning techniques. Cereb. Cortex 25, 3046-3056. doi: 10 . 1093/cercor/bhu100

Rilling, J. K., Glasser, M. F., Preuss, T. M., Ma, X., Zhao, T., Hu, X., et al. (2008). The evolution of the arcuate fasciculus revealed with comparative DTI. Nat. Neurosci. 11, 426-428. doi: 10.1038/nn2072

Sanford, J., Moreland, J., Swanson, L. R., Stratford, P. W., and Gowland, C. (1993). Reliability of the Fugl-Meyer assessment for testing motor performance in patients following stroke. Phys. Ther. 73, 447-454.

Schulz, R., Park, C.-H., Boudrias, M.-H., Gerloff, C., Hummel, F. C., and Ward, N. S. (2012). Assessing the integrity of corticospinal pathways from primary and secondary cortical motor areas after stroke. Stroke 43, 2248-2251. doi: 10. 1161/STROKEAHA.112.662619

Smania, N., Paolucci, S., Tinazzi, M., Borghero, A., Manganotti, P., Fiaschi, A., et al. (2007). Active finger extension: a simple movement predicting recovery of arm function in patients with acute stroke. Stroke 38, 1088-1090. doi: 10. 1161/01.str.0000258077.88064.a3

Sotak, C. H. (2002). The role of diffusion tensor imaging in the evaluation of ischemic brain injury-a review. NMR Biomed. 15, 561-569. doi: 10.1002/ nbm.786

Stinear, C., Ackerley, S., and Byblow, W. (2013). Rehabilitation is initiated early after stroke, but most motor rehabilitation trials are not: a systematic review. Stroke 44, 2039-2045. doi: 10.1161/STROKEAHA.113. 000968

Stinear, C. M., Barber, P. A., Petoe, M., Anwar, S., and Byblow, W. D. (2012). The PREP algorithm predicts potential for upper limb recovery after stroke. Brain 135, 2527-2535. doi: 10.1093/brain/aws 146

Stinear, C. M., Byblow, W. D., and Ward, S. H. (2014). An update on predicting motor recovery after stroke. Ann. Phys. Rehabil. Med. 57, 489-498. doi: 10. 1016/j.rehab.2014.08.006

Urbin, M. A., Hong, X., Lang, C. E., and Carter, A. R. (2014). Resting-state functional connectivity and its association with multiple domains of upperextremity function in chronic stroke. Neurorehabil. Neural Repair 28, 761-769. doi: $10.1177 / 1545968314522349$

van Dokkum, L., Hauret, I., Mottet, D., Froger, J., Métrot, J., and Laffont, I. (2014). The contribution of kinematics in the assessment of upper limb motor recovery early after stroke. Neurorehabil. Neural Repair 28, 4-12. doi: 10. $1177 / 1545968313498514$

Wang, L., Yu, C., Chen, H., Qin, W., He, Y., Fan, F., et al. (2010). Dynamic functional reorganization of the motor execution network after stroke. Brain 133, 1224-1238. doi: 10.1093/brain/awq043

World Health Organization. (2012). The 10 Leading Causes of Death in the World. Geneva: World Health Organization.

Yousry, T. A., Schmid, U. D., Alkadhi, H., Schmidt, D., Peraud, A., Buettner, A., et al. (1997). Localization of the motor hand area to a knob on the precentral gyrus. A new landmark. Brain 120, 141-157. doi: 10.1093/brain/120. 1.141

Conflict of Interest Statement: The authors declare that the research was conducted in the absence of any commercial or financial relationships that could be construed as a potential conflict of interest.

Copyright (c) 2016 Lindow, Domin, Grothe, Horn, Eickhoff and Lotze. This is an open-access article distributed under the terms of the Creative Commons Attribution License (CC BY). The use, distribution and reproduction in other forums is permitted, provided the original author(s) or licensor are credited and that the original publication in this journal is cited, in accordance with accepted academic practice. No use, distribution or reproduction is permitted which does not comply with these terms. 